\title{
The Best Treatment of Stroke Is Prevention!
}

Preventing stroke is the topic priority for all of us. $S V N$ is grateful that our Guest Chief Editor for the June issue, Professor J. David Spence, has organised several important manuscripts on stroke prevention. Professor Spence has dedicated his entire career to stroke prevention. He opened the third hypertension clinic in Canada, pioneered the measurement of 2-dimensional carotid total plaque, and developed a new paradigm for vascular prevention- "treating arteries instead of treating risk factors," which has markedly reduced risk among high-risk patients with carotid stenosis. He is also an expert on vitamin therapy for homocysteine and physiologically individualized therapy for resistant hypertension.

In this special issue, 9 articles cover topics that are important and practical to stroke practice and patient care. Updated information and current thoughts were provided by the authorities of the world on these topics: diet, exercise, blood pressure control, PFO, vitamins, antiplatelet agents, anticoagulation, and carotid disease. Furthermore,the meta-analysis on the use of antiplatelet drugs will give readers a cutting edge overview on this topic since the publication of the POINT trial. We are confident that this special issue will provide useful information that will apply to healthcare providers and researchers in the field of cerebral vascular disease and stroke.

As one of the newest subspecialty journals in cerebral vascular diseases, $S V N$ is committed to bring timely new and updated scientific discoveries and reviews on stroke to the world. 